\title{
A High-Order Nodal Discontinuous Galerkin Method for 1D Morphodynamic Modelling
}

\author{
Nouh Izem \\ EMMS Faculty of science, Ibn \\ Zohr University Agadir, \\ Morocco
}

\author{
Mohammed Seaid \\ School of Engineering and \\ Computing Sciences, University \\ of Durham, UK
}

\author{
Mohamed Wakrim \\ EMMS Faculty of science, Ibn \\ Zohr University Agadir, \\ Morocco
}

\begin{abstract}
In this paper we present a numerical solution of the sediment transport equations in one horizontal dimension, based on a discontinuous Galerkin finite-element method. The continuous equations are discretized using nodal polynomial basis functions of arbitrary order in space on each element of an unstructured computational domain. To complete the discretization in space, we choose the numerical flux based in the local Lax-Friedrichs flux. A third-order explicit RungeKutta scheme is used to advance the solution in time. In spite of the local time steps the scheme is locally conservative, fully explicit, and arbitrary order accurate in space and time for transient calculations. Numerical results are shown for the one-dimensional with orders of accuracy two up to six in space.
\end{abstract}

\section{Keywords}

Morphodynamic model; Discontinuous Galerkin finiteelement method; Shallow water equations; Sediment transport

\section{INTRODUCTION}

During the last decades, new engineering problems, connected with gravel extraction, installation of dams and disposal of mine waste into rivers, have risen to the extent that sediment transport modeling is assuming a key role in realistic river hydraulic simulations. Floods, meandering, sediment load computation, river bed aggradation or degradation, channel design and navigation are some of the problems concerning the sediment transport in rivers.

In literature, different models have been proposed in order to quantify the interaction between sediment transport and water flow. To progress on quantifying such interactions, it becomes necessary to develop numerical methods that accurately simulate the fluid flow over a movable bed. Generally, the mathematical modeling of morphodynamic relies on a system of three equations: two equations (shallow water equations) describing flow continuity and momentum conservation with shallow water assumptions, and one equation (Exner equation) expressing the sediment continuity. The numerical solution of the resulting system of equations is nontrivial and two basic approaches have been adopted as a common practice, namely, the coupled and decoupled approach $[16,11$, 14]. The conventional method for performing morphodynamic simulations at coasts and in rivers is to decouple the hydrodynamic and the morphodynamic systems. In the decoupled approach [16] the hydrodynamic and morphodynamic equations are discretized separately assuming that the water motion is steady with respect to changes in the bed level. For the coupled approach, no assumptions are made and the water flow and bed are calculated simultaneously. With this approach, the water motions can either be steady or unsteady and the changes in the bed update are considered to be significant. Here the wave speed of the sediment continuity equation can be of a similar magnitude to the wave speeds of the water flow. For this approach, the system is discretised simultaneously.

Several numerical methods have been successfully applied to solve the sediment transport equations. In [12], the authors modified the well-established Roe's scheme [20]. An interesting approach based on Euler-WENO techniques have also been applied to one-dimensional sediment transport equations in [18]. On the other hand, authors in [19] extended the ENO and WENO schemes to one-dimensional sediment transport equations, whereas the CWENO method has been applied to sediment transport problems in [3]. In the framework of kinetic schemes, a method based on relaxation approach have also been applied to sediment transport equations in [6]. The emphasis of the present work is on the application of the so-called nodal Discontinuous Galerkin (DG) methods to the sediment transport problems. The nodal DG method first introduced by Hesthaven and Warburton [10] for electro-dynamic simulations utilizes a nodal Lagrange interpolation basis as the approximating basis functions, which provides a simple and generic means to treat a (nonlinear) flux term appearing in the hyperbolic conservation laws. The main features of the proposed DG method are: (i) the capability to preserve the advantages of the classical finite element method to handle complicated geometries, (ii) the incorporation of mesh adaptivity without requiring the continuity assumptions typical of conforming finite element methods, (iii) the ability to increase the overall accuracy by increasing the degree of the approximating polynomials locally, thus allowing an efficient $p$ adaptivity for each element independently of its neighbors (iv) the efficient parallel implementation, since each element solution communicates with its immediate neighbors only, regardless of the order of accuracy, and (v) the ability to handle calculations of slowly varying flows as well as rapidly varying flows over continuous and discontinuous bottom beds. Some of these features are demonstrated using several test problems for sediment transport flows. Results presented in this paper show high resolution of the proposed DG method and confirm its capability to provide robust and accurate 
simulations for sediment transport problems including complex topography.

The structure of the paper is organized as follows. Section 2 reviews the governing sediment transport equations to be solved. Section 3 describes the numerical scheme and discusses in detail the spatial discretization of the solution as well as the temporal discretization used to advance the solution in time with descriptions of the limiting techniques that is necessary to stabilize the nonlinear calculations. Section 4 we demonstrate the accuracy, efficiency, and robustness of the complete scheme for the solution of sediment transport equations the results is compared to SRNHS finite volume method $[8,7]$.

\section{MATHEMATICAL MODEL}

In one space dimension, the sediment transport is usually modelled by the shallow water equations. These equations consist of the conservation of mass and momentum balance:

$$
\begin{aligned}
& \partial_{t} h+\partial_{x}(h u)=0, \\
& \partial_{t}(h u)+\partial_{x}\left(h u^{2}+\frac{1}{2} g h^{2}\right)=-g h \partial_{x} Z,
\end{aligned}
$$

where $h$ is the water height above the bottom, $u$ is the water velocity, $g$ is the acceleration due to gravity, and $Z$ is the bed evaluation. When the sediment transport phenomenon occurs, the bed level becomes a function of the time variable too and the equation that describes the bed-load sediment transport must be added

$$
\partial_{t} Z+\xi \partial_{x} q=0
$$

Where

$$
\xi=\frac{1}{1-\varepsilon}
$$

and $\varepsilon$ being the porosity of the riverbed, and it depends on the type of the sediment. In equation (2), $q$ is the total volumetric sediment transport rate in the $\mathrm{x}$-direction, i.e., the sediment transport flux. One possible mathematical model for the sediment transport flux is the simple law [1].

$$
\mathrm{q}(\mathrm{u})=A u|u|^{m-1}
$$

Where $A$ is a given experimental constant and $1 \leq m \leq 4$ is a chosen parameter. We should mention that the DG method described in this paper can be applied to other forms of sediment transport fluxes (see for instance [21, 22]) without major conceptual modifications. If $A=0$ then we have a solid bed (no sediment transport) and we recover the standard shallow water equations. When $A$ is near zero there is a small interaction between the fluid and the bed, while if $A$ is near one the interaction is larger. Under these assumptions, the sediment transport problem can be rewritten in a conservative form as

$$
\partial_{t} W+\partial_{x} F(W)=Q(W),
$$

Where $W, F(W)$ and $Q$ are the vector-valued functions in $I R^{3}$ given by

$$
W=\left(\begin{array}{c}
h \\
h u \\
Z
\end{array}\right), \quad F(W)=\left(\begin{array}{c}
h u \\
h u^{2}+\frac{1}{2} g h^{2} \\
\xi q
\end{array}\right), \quad Q=\left(\begin{array}{c}
0 \\
-g h \partial_{x} Z \\
0
\end{array}\right)
$$

In what follows we discuss the formulation of the DG method for solving the morphodynamic system depending on the values taken by $A$.

\subsection{Coupled approach}

In the test case where $A=1 s^{2} / m$, the changes in the bed update are considered to be significant. Here the wave speed of the sediment continuity equation can be of a similar magnitude to the wave speeds of the water flow i.e. high intensity bed load rates are considered and the time scales of bed evolution and hydrodynamics are comparable. Therefore the fully coupled problem must be solved. For this purpose, the sediment transport equations can also be reformulated in a nonconservative system as

$$
\partial_{t} U_{1}+\partial_{x} G_{1}\left(U_{1}\right)=0
$$

Where

$$
U_{1}=\left(\begin{array}{l}
h \\
u \\
B
\end{array}\right),
$$$$
G_{1}\left(U_{1}\right)=\left(\begin{array}{c}
h u \\
\frac{1}{2} u^{2}+g(h+Z) \\
\xi q
\end{array}\right),
$$

Note that the above system is obtained from a modification of the equations in the original sediment transport equations (1) and (2) in order to avoid the singularity in the Jacobian matrix of the flux function $F$. It is clear that

$$
\nabla G_{1}\left(U_{1}\right)=\left(\begin{array}{lll}
u & h & 0 \\
g & u & g \\
0 & d & 0
\end{array}\right),
$$

Where $d=A \xi_{m}|u|^{m-1}$, Furthermore, since the system (4) is hyperbolic, we can rewrite

$$
\nabla G_{1}=R \Lambda R^{-1}
$$

With $R$ is the matrix of right eigenvectors of $\nabla G_{1}$ and $\Lambda$ is a diagonal matrix with eigenvalues of $\nabla G_{1}$ as its elements. The eigenvalues of $\nabla G_{\mathrm{P}}, \lambda_{k}(k=1,2,3)$ are the zeros of the characteristic polynomial

$$
P\left(\lambda, U_{1}\right)=\lambda^{3}-2 u \lambda^{2}+\left(u^{2}-g(h+d)\right) \lambda+g u d .
$$

With

$$
d=\frac{1}{(1-\varepsilon)} A m|u|^{m-1}
$$

Thus, the eigenvalues of the Jacobian matrix are

$$
\begin{aligned}
& \begin{aligned}
\lambda_{1} & =2 \sqrt{-Q} \cos \left(\frac{1}{3} \theta\right)+\frac{2}{3} u, \\
\lambda_{2} & =2 \sqrt{-Q} \cos \left(\frac{1}{3}(\theta+2 \pi)\right)+\frac{2}{3} u, \\
\lambda_{3} & =2 \sqrt{-Q} \cos \left(\frac{1}{3}(\theta+4 \pi)\right)+\frac{2}{3} u,
\end{aligned} \\
& \text { Where } \theta=\operatorname{arcos}\left(\frac{R}{\sqrt{-Q^{3}}}\right) \text { with }
\end{aligned}
$$


$Q=\frac{1}{9}\left(u^{2}+3 g(h+d)\right), \quad R=\frac{u}{54}\left(9 g(2 h-d)-2 u^{2}\right)$.

The corresponding eigenvectors are

$$
e_{k}=\left(\begin{array}{c}
1 \\
\frac{\lambda_{k}-u}{h} \\
\frac{\left(u-\lambda_{k}\right)^{2}-g h}{g h}
\end{array}\right), \quad k=1,2,3
$$

It should be stressed that the analytical expressions of eigenvalues $\lambda_{k}$ can be explicitly calculated and for details we refer the reader to $[12,11]$.

\subsection{Decoupled approach}

In this case, we set $A$ equal to $0.001 \mathrm{~s}^{2} / \mathrm{m}$. The moderate bed load sediment transport allows us to consider the bed as fixed with respect to the hydrodynamics within a time step. These assumptions allow the water flow to be discretised separately from the bed. Moreover, the approach takes advantage of the slow evolution of the bed by iterating the water flow,

$$
\partial_{t} U_{2}+\partial_{x} G_{2}\left(U_{2}\right)=0
$$

Where

$$
U_{2}=\left(\begin{array}{l}
h \\
u
\end{array}\right), \quad G_{2}\left(U_{2}\right)=\left(\begin{array}{c}
h u \\
\frac{1}{2} u^{2}+g(h+Z)
\end{array}\right),
$$

to an equilibrium state each time the bed is updated. Since the ratio between bed load sediment transport in the previous approach and in the present approach is 1000 , bed elevation is updated one time each 1000 computational steps. This is completely equivalent to choose a different time scaling for the third equation of system (1) -(2) with respect to the other set of equations. In these computations, we have chosen

$$
\Delta t_{\text {bed }}^{n}=\sum_{k=1}^{1000} \Delta t_{\text {hydrodynarics }}^{n-1000+k}
$$

This option reduces the numerical diffusion generated by the staggering procedure. Such numerical diffusion may affect bed elevation when a slow bed evolution occurs and a long computational time must be considered. For this approach, the Jacobian matrix of (6) is

$$
\nabla G_{2}\left(U_{2}\right)=\left(\begin{array}{cc}
0 & 1 \\
c^{2}-u^{2} & u
\end{array}\right), \quad c=\sqrt{g h},
$$

whose eigenvalues are

$$
\lambda_{1}=u-c, \quad \lambda_{2}=u+c,
$$

with corresponding eigenvectors

$$
e_{1}=\left(1, \lambda_{1}\right)^{T}, \quad e_{2}=\left(1, \lambda_{2}\right)^{T}
$$

Remarque 2.1 It is important to notice here that when considering the sediment transport equation (1) - (2) the inhomogeneous right hand side terms in (1) are not standard source terms but nonconservative products, since they include a derivative of one of the variables. This fact can cause difficulties in the numerical approximations. However, both formulations (4) and (6) adopted here avoids this problem by incorporating the source term in the flux function.

\section{A DISCONTINUOUS GALERKIN METHOD}

The different approaches (4) - (6) discussed in the previous section can be written in the general form as

$$
\frac{\partial U}{\partial t}+\frac{\partial G(U)}{\partial x}=0
$$

where $U$ can be either $U_{1}$ or $U_{2}$ and $G$ being $G_{1}$ or $G_{2}$. In this section, we first discuss a discontinuous Galerkin finite element method for the spatial discretization. Then a thirdorder Runge-Kutta time integration scheme is formulated for the semi-discrete system.

\subsection{The Spacial Discretisation}

For simplicity in the presentation we discretize the computational domain $\Omega$ into $M$ non-overlapping elements, $\Omega_{k}=\left[x_{k}\right.$ $\left.{ }_{1 / 2}, x_{k+1 / 2}\right], k=1, \ldots, M$, with mesh size $\Delta x_{k}=x_{k+1 / 2}-x_{k-1 / 2}$ such that $\Omega=\bigcup_{k=1}^{M} \Omega_{k}$ We also denote $\Delta x=\max _{k} \Delta x_{k}$. We assume that the global solution $U$ of (8) on the computational domain can be represented as a direct sum of local piecewise polynomial solutions as

$$
U(x) \cong U_{h}(x)=\bigoplus_{k=1}^{M} U_{h}^{k}(x),
$$

where the solution is locally approximated on the element $\Omega_{k}$ by a finite sum as

$$
U^{k}(x) \cong U_{h}^{k}(x)=\sum_{n=0}^{N} \widehat{U}_{n}^{k} \phi_{n}(x)=\sum_{n=0}^{N} U_{h}^{k} l_{n}(x),
$$

where we have $(N+1)$ degrees of freedom inside each element in terms of the unknown modal $\widehat{U}_{n}^{k}$ coefficients or nodal coefficients $U_{h}^{k}$ for $n=0,1, \ldots, N . \quad l_{n}(x)$ are the Lagrange polynomials defined on the set of nodes used in combination with the chosen orthogonal basis. The basis functions $\phi_{n}$, with $n=0,1, \ldots, N$, are chosen such that $U^{k} \in V_{k}^{N}$, where the approximation space is defined as

$$
V_{k}^{N}=\left\{v: v_{k} \in I P^{N}\left(\Omega_{k}\right), \forall \Omega_{k} \in \Omega\right\}
$$

where $I P^{\mathrm{N}}\left(\Omega_{k}\right)$ denotes the set of polynomials of degree up to $N$ defined on the element $\Omega_{k}$. As orthogonal basis, we consider the classical Legendre polynomials $P_{n}(x)$ such that

$$
\phi_{n}(x)=\frac{P_{n}(x)}{\sqrt{2 n+1}} .
$$

As shown in [10] to represent the polynomial basis functions in a Lagrangian (nodal) basis, we use the Legendre-GaussLobatto nodes. By uniqueness of the interpolation polynomials, the following (constant) transformation matrix can be defined for transforming the variables between the modal and nodal spaces

$$
V_{i j}=\phi_{j}\left(x_{i}\right), \quad i, j=0,1, \ldots, N .
$$

Here, $x_{i}$ is the $i$ th nodal point inside the $k$ th element $\Omega_{k}$. This yields the following relationship between the modal and nodal coefficients and basis functions

$$
U_{k}=V \tilde{U}^{k}, \quad \phi_{i}(x)=V^{T} l_{i}(x) .
$$

Note that the relation (10) allows us to determine both the mass matrix and the stiffness matrix exactly for a given 
element without using high-order Gaussian quadrature rules for the element integrals.

Applied to the system (8), we obtain the following polynomial representations of the local solution $U_{h}^{k}$ and the local flux function $G_{h}^{k}$

$$
U_{h}^{k}(x, t)=\sum_{n=0}^{N} U_{n}^{k} l_{i}^{k}\left(x_{i}, t\right), \quad G_{h}^{k}\left(U_{h}(x, t)\right)=\sum_{n=0}^{N} G^{k}\left(x_{i}, t\right) l_{i}^{k}(x),
$$

It should be pointed out that we assume that the local residual to be orthogonal to all test functions $\phi_{n} \in V_{k}^{N}$. Thus, after integrating by parts, we get the local semi-discrete weak formulation

$$
\int_{\Omega_{k}}\left(\frac{\partial U_{h}^{k}}{\partial t} l_{i}^{k}(x)-G_{h}^{k}\left(U_{h}^{k}\right) \frac{d l_{n}^{k}}{d x}\right) d x,=-\int_{\partial \Omega_{k}} \hat{n} \cdot G^{*} l_{i}^{k}(x) d x,
$$

and the corresponding strong form

$$
\int_{\Omega_{k}}\left(\frac{\partial U_{h}^{k}}{\partial t}+\frac{\partial G_{h}^{k}\left(U_{h}^{k}\right)}{\partial x}\right) l_{i}^{k}(x) d x,=-\int_{\partial \Omega_{k}} \hat{n} \cdot\left(G_{h}^{k}\left(U_{n}^{k}\right)-G^{*}\right) l_{i}^{k}(x) d x,
$$

where $\hat{n}$ denotes the unit normal vector on $\partial \Omega_{k}$ and $G_{k}$ is a monotone numerical flux associated with $G$. In the present work, we consider the local monotone Lax-Friedrichs flux defined by [17]

$$
G^{*}\left(U_{h}^{-}, U_{h}^{+}\right)=\frac{1}{2}\left(G\left(U_{h}^{-}\right)+G\left(U_{h}^{+}\right)\right)-\frac{1}{2} \lambda \hat{n} \cdot\left(U_{h}^{-}-U_{h}^{+}\right),
$$

where $U_{h}^{-}$and $U_{h}^{+}$are respectively, the left and right limits of $U$ at the interface where $U$ is discontinuous, and

$$
\lambda=\max _{k}\left(\left|\lambda_{k}\right|\right)
$$

With $\lambda_{k}$ are the approximated eigenvalues in (5) or (7) according to the considered approach.

The local monotone Lax-Friedrichs numerical flux is a particularly convenient choice of numerical flux because it can be easily applied to any non-linear hyperbolic system, it is simple to compute, and yields good results, although there are many other numerical fluxes which could also be used [15].

From the strong formulation (12) we can generate a local linear system of the form

$$
M^{k} \frac{d U_{h}^{k}}{d t}+S G_{h}^{k}=\left[l^{k}(x)\left(G_{h}^{k}-G^{*}\right)\right]_{x_{k-1 / 2}}^{x_{k+1 / 2}},
$$

where $G_{h}^{k}=\left(G_{h}^{k}\left(x_{0}^{k}\right), \ldots, G_{h}^{k}\left(x_{N}^{k}\right)\right)^{T}, U_{h}^{k}=\left(U_{h}^{k}\left(x_{0}^{k}\right), \ldots, U_{h}^{k}\left(x_{N}^{k}\right)\right)^{T}$ and $G^{*}$ is the vector of the associated numerical fluxes in (13). The mass matrix $M_{i j}^{k}$ and the stiffness matrix $S_{i j}^{k}$ on $\Omega_{k}$ are

$$
\begin{aligned}
M_{i j}^{k} & =\int_{\Omega_{k}} l_{i}^{k}(x) l_{j}^{k}(x) d x, \\
S_{i j}^{k} & =\int_{\Omega_{k}} l_{i}^{k}(x) \frac{d l_{n}^{k}}{d x} d x,
\end{aligned}
$$

To compute the mass matrix, we introduce an affine transformation defined as

$$
x \in \Omega_{k}: x(r)=x_{k+1 / 2}+\frac{1+r}{2} \Delta x_{k},
$$

with the reference variable $r \in I=[-1,1]$. Hence,

$$
M_{i j}^{k}=\frac{\Delta x_{k}}{2} \int_{-1}^{1} l_{i}(r) l_{j}(r) d r=\frac{\Delta x_{k}}{2} M_{i j}
$$

where the coefficient $\frac{\Delta x_{k}}{2}$ comes from the Jacobian in (16) and is the mass matrix $M^{2}$ defined on the reference element $I$. Recall that

$$
l_{i}(r)=\sum_{n=1}^{N+1}\left(V^{T}\right)_{i n}^{-1} \hat{P}_{n-1}(r)
$$

from which we recover

$$
\begin{aligned}
M_{i j} & =\int_{-1}^{1} \sum_{n=1}^{N+1}\left(V^{T}\right)_{i n}^{-1} \hat{P}_{n-1}(r) \sum_{m=1}^{N+1}\left(V^{T}\right)_{i m}^{-1} \hat{P}_{m-1}(r) d r, \\
& =\sum_{n, m=1}^{N+1}\left(V^{T}\right)_{i n}^{-1}\left(V^{T}\right)_{j m}^{-1} \int_{-1}^{1} \hat{P}_{n-1}(r) \hat{P}_{m-1}(r) d r, \\
& =\sum_{n=1}^{N+1}\left(V^{T}\right)_{i n}^{-1}\left(V^{T}\right)_{j n}^{-1}
\end{aligned}
$$

where the latter follows from the orthogonality of $\hat{P}_{n}(r)$. Thus,

$$
M^{k}=\frac{\Delta x_{k}}{2} M=\frac{\Delta x_{k}}{2}\left(V V^{T}\right)^{-1}
$$

Similarly, the stiffness matrix $S^{k}$ is given as

$$
S_{i j}^{k}=\frac{\Delta x_{k}}{2} \int_{-1}^{1} l_{i}(r) \frac{d l_{j}(r)}{d r} d r=S_{i j}
$$

To calculate the derivative in (17), we define the differentiation matrix $D_{\mathrm{r}}$ the entries of which are given by

$$
D_{r, i j}=\left.\frac{d l_{j}}{d r}\right|_{r_{i}} \text {. }
$$

Consider now the product $M D_{\mathrm{r}}$ the entries of which are defined as

$$
\left(M D_{r}\right)_{i j}=\left.\sum_{n=1}^{N+1} \int_{-1}^{1} l_{i}(r) l_{n}(r) \frac{d l_{j}(r)}{d r}\right|_{r_{n}} d r=\left.\int_{-1}^{1} l_{i}(r) \frac{d l_{j}(r)}{d r}\right|_{r_{n}} d r=S_{i j}
$$

which can be rewritten in a matrix form as

$$
M D_{r}=S \text {. }
$$

The entries of the differentiation matrix $D_{r}$ can be found directly as

$$
V^{T} l(r)=\hat{P}(r) \quad \Rightarrow \quad V^{T} \frac{d l(r)}{d r}=\frac{d \hat{P}(r)}{d r},
$$

leading to

$$
V^{T} D_{r}^{T}=V_{r}^{T}, \text { where } V_{r, i j}=\left.\frac{d \hat{P}_{j}}{d r}\right|_{r_{i}} .
$$

Finally, we obtain

$$
M^{k}=\frac{\Delta x_{k}}{2} M=\frac{\Delta x_{k}}{2}\left(V V^{T}\right)^{-1}, \quad S=M D_{r},
$$

Thus, the semi-discrete equations (15) reduce to

$$
\frac{\Delta x_{k}}{2} M \frac{d U_{h}^{k}}{d t}+M D_{r} G_{h}^{k}=\left[l^{k}(x)\left(G_{h}^{k}-G^{*}\right)\right]_{x_{k-1 / 2}}^{x_{k+1 / 2}}
$$

It should be stressed that boundary conditions have to be incorporated in the semi-discrete system (19). In the DG framework it is usual to impose boundary conditions in a weak form in both, inflow and outflow, boundaries. It is recognized for most works published in the literature, that the weak imposition of Dirichlet-type conditions is superior to the strong imposition on outflow boundaries, see for instance [2]. This is due to the appearance of spurious oscillations in boundary layers when Dirichlet boundary conditions are imposed strongly. However, the weak enforcement of inflow 
Dirichlet boundary conditions offers no advantages over the strong imposition, compare for example [13] and further discussions are therein.

\subsection{The time integration}

The solution procedure for equations (8) is complete when a time integration of semi-discrete equations (19) is selected. This stage can be handled by any implicit ordinary differential equations (ODE) solver, since they are computationally without risk by virtue of their accuracy and linear unconditionally stability. This allows for larger time steps in the integration process. However, due to the large set of linear system of algebraic equations at each time step, these methods may be computationally inefficient. As an alternative, we use an explicit Runge-Kutta method studied in [23]. By assembling together all the elemental contributions, the system (19) can be written as

$$
\frac{d U_{h}^{k}}{d t}=-\frac{2 D_{r}}{\Delta x_{k}} G_{h}^{k}+\frac{2}{\Delta x_{k}} M^{-1}\left[l^{k}(x)\left(G_{h}^{k}-G^{*}\right)\right]_{x_{k-1 / 2}}^{x_{k+1 / 2}} .
$$

Let us rewrite the equations (20) in a compact ODE form as

$$
\begin{aligned}
& \frac{d U}{d t}=L(U), \quad t \in(0, T), \\
& U(0)=U_{0},
\end{aligned}
$$

where $L$ represents the right-hand side in (20) and $U_{0}$ is a given initial data. The procedure to advance the solution from the time $t_{\mathrm{n}}$ to the next time $t_{\mathrm{n}+1}$ can be carried out as

$$
\begin{aligned}
U^{(1)} & =U^{n}+\Delta t L\left(U^{n}\right) \\
U^{(2)} & =\frac{3}{4} U^{n}+\frac{1}{4} U^{(1)}+\frac{1}{4} \Delta t L\left(U^{(1)}\right) \\
U^{n+1} & =\frac{1}{3} U^{n}+\frac{2}{3} U^{(2)}+\frac{2}{3} \Delta t L\left(U^{(2)}\right) .
\end{aligned}
$$

This class of explicit time integration schemes has become popular in computational fluid dynamics, see for example [9]. The main feature of this method lies on the fact that (22) is a convex combination of first-order Euler steps which exhibits strong stability properties. Therefore, the scheme (22) is TVD, third-order accurate in time, and stable under the usual Courant-Friedrichs-Lewy (CFL) condition

$$
\lambda \frac{\Delta t}{\Delta x} \leq 1
$$

where $\lambda$ is defined in (14). In order to prevent non-physical oscillations and to obtain high-order stability for the space discretization $k \geq 1$, a slope limiter is applied on every solution in the Runge-Kutta stages (22). in the current study, to modify the local mean of the solution to guarantee the TVD property in the mean, we first consider the following minmod function

$$
\operatorname{minmod}\left(v_{1}, v_{2}, \ldots, v_{N}\right)=\left\{\begin{array}{ll}
s \min _{1 \leq i \leq N}\left|v_{i}\right|, & \text { if }|s|=1, \\
0, & \text { otherwise, }
\end{array} \quad s=\frac{1}{N} \sum_{i=1}^{N} \operatorname{sign}\left(v_{i}\right) .\right.
$$

Then, we define the interface fluxes as

$$
\begin{aligned}
& W_{l}^{k}=\bar{U}_{h}^{k}-\operatorname{minmod}\left(\bar{U}_{h}^{k}-U_{l}^{k}, \bar{U}_{h}^{k}-\bar{U}_{h}^{k-1}, \bar{U}_{h}^{k+1}-\bar{U}_{h}^{k}\right), \\
& W_{r}^{k}=\bar{U}_{h}^{k}-\operatorname{minmod}\left(\bar{U}_{r}^{k}-U_{h}^{k}, \bar{U}_{h}^{k}-\bar{U}_{h}^{k-1}, \bar{U}_{h}^{k+1}-\bar{U}_{h}^{k}\right) .
\end{aligned}
$$

We assume that the solution is represented by a piecewise linear function as

$$
U_{h}^{k}(x)=\bar{U}_{h}^{k}+\left(x-x_{0}^{k}\right)\left(U_{h}^{k}\right)_{x},
$$

Where $\left(\boldsymbol{U}_{h}^{k}\right)_{x}$ denotes the derivative of $\boldsymbol{U}_{h}^{k}$ with respect to $x$ and $x_{0}$ represents the center point in the element $\Omega_{k}$. Hence, the slope limited solution using the MUSCL function [17] is given by

$$
\prod U_{h}^{k}(x)=\bar{U}_{h}^{k}+\left(x-x_{0}^{k}\right) \operatorname{minmod}\left(\left(U_{h}^{k}\right)_{x}, \frac{\bar{U}_{h}^{k+1}-\bar{U}_{h}^{k}}{\Delta x_{k}}, \frac{\bar{U}_{h}^{k}-\bar{U}_{h}^{k-1}}{\Delta x_{k}}\right) .
$$

It well-known that this procedure may reduce the order of the approximation in the vicinity of the sharp front if an oscillation is detected. A simple way to avoid this drawback is to apply the limiters only in those elements where oscillations are detected, see for example [4]. This procedure can be implemented in the following steps:

1. Compute the limited edge values $W_{l}^{k}$ and $W_{r}^{k}$ using the equations (25).

2. If $\boldsymbol{W}_{l}^{k}=\boldsymbol{U}_{h}^{k}\left(x_{l}^{k}\right)$ and $\boldsymbol{W}_{r}^{k}=\boldsymbol{U}_{h}^{k}\left(x_{r}^{k}\right)$, there is no need for limiting and the local solution is not altered.

3. If a limiting process is needed, compute the limited version of $\boldsymbol{U}_{h}^{k}$ as $\prod \hat{U}_{h}^{k}$, where is the linear approximation to given by (26).

Note that other slope limiter functions can also be applied in (27).

\section{NUMERICAL RESULTS}

In this section we present numerical results obtained for several test examples on sediment transport equations in one space dimension. All the test examples use the sediment transport flux (3) with $m=3$, the space domain is discretized in 400 gridpoints and a fixed courant number $C r=0.75$ is used while the time step is varied according to the stability condition

$$
\Delta t=C r \frac{\Delta x}{\max _{k}\left(\left|\lambda_{k}\right|\right)},
$$

For comparison reasons, we have included a solution computed by the first-order SRNH scheme and the secondorder SRNHS scheme using 400 grid points as such we compare the results produced by the proposed DG-scheme with those obtained by SRNHS finite volume method $[8,7]$.

For all the problems that we will present here we consider the channel length to be $1000 \mathrm{~m}$, treating the boundary points using free flow boundary conditions. Using variations of physical parameters such as $A, \varepsilon$ and $Q$ we can obtain different problems. Initial water level and initial velocity are given as

$$
h(0, x)=10-Z(0, x) \text { and } u(0, x)=\frac{Q}{h(0, x)},
$$

where $Q$ is a constant discharge taken the value $10 \mathrm{~m}^{2} / \mathrm{s}$. Two values $A=0.001$ and $A=1$ are used in the the sediment transport flux (3).

\subsection{Test 1}

We consider test example studied in [7]. The porosity coefficient is $\varepsilon=0.4$ and the initial bed is defined as

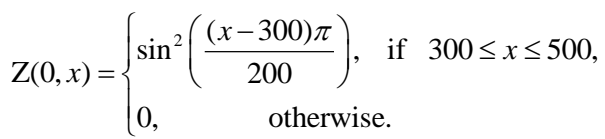



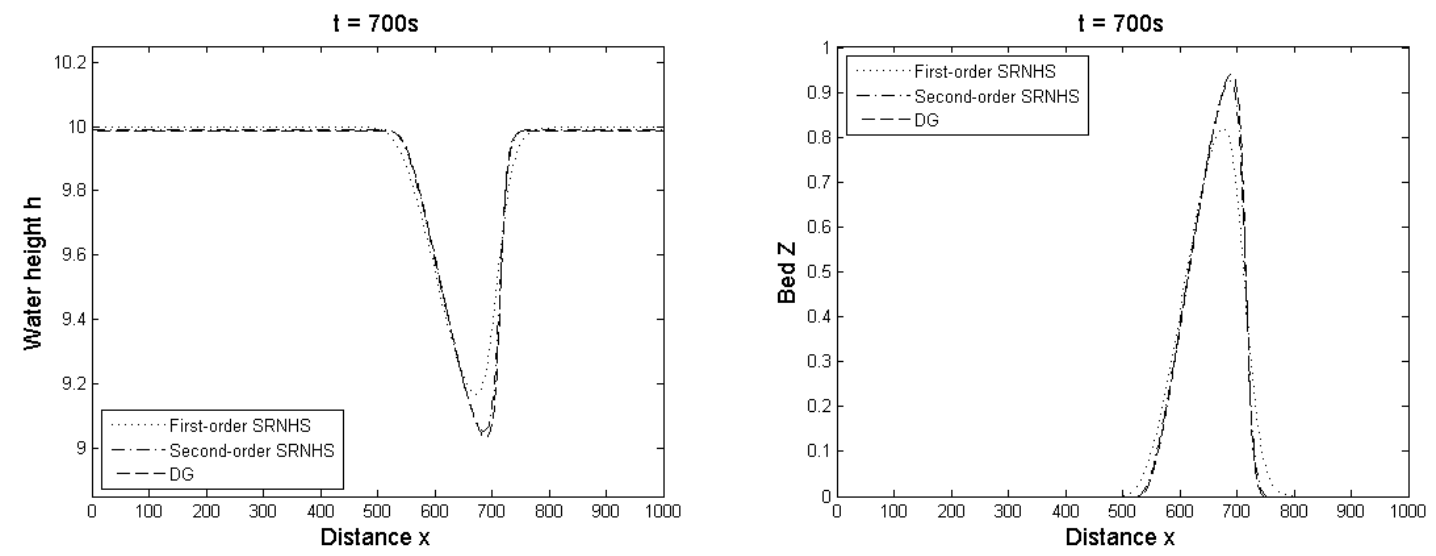

Fig 1: Water height (left) and bed-load (right) with $A=1$ using coupled approach.
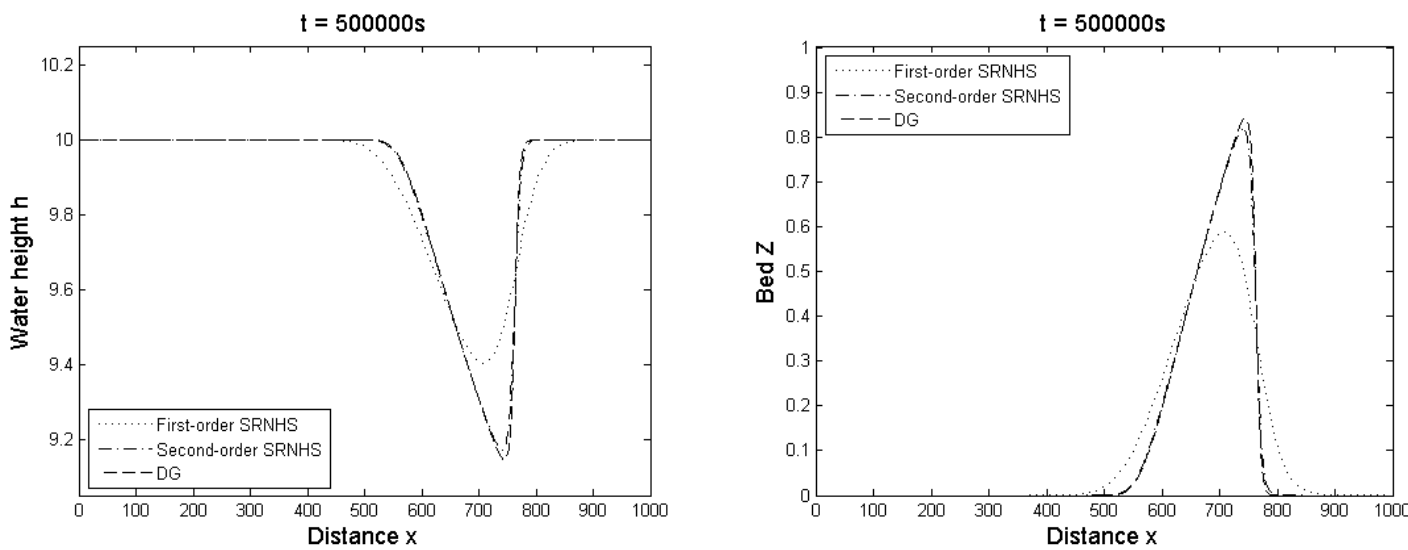

Fig 2: Water height (left) and bed-load (right) with $A=0.001$ using decoupled approach.

We first present in Figure 1 the obtained results using $A=1$. Next we display in Figure 2 results obtained with $A=0.001$ using $Q=10 \mathrm{~m}^{2} / \mathrm{s}$. It is clear that the first-order SRNHS scheme produces diffusive Mobil-bed and water level resulting in smearing the shocks. However, this numerical diffusion has remarkably be reduced in the results computed using the second-order SRNHS scheme and more better using the DG scheme. The downstream propagation of the wave is well-reproduced by the DG method using a piecewise cubic polynomials. The total absence of spurious oscillations and the numerical comparison $[6,8]$ support this statement.

\subsection{Test 2}

This test problem considers the evolution of an initially discontinuous bed in a channel proposed in $[8,6]$. Due to this discontinuity in the initial bed profile, this test example is more challenging than the previous example. The initial bed is defined as

$$
Z(0, x)= \begin{cases}1, & \text { if } 300 \leq x \leq 500, \\ 0, & \text { otherwise. }\end{cases}
$$

The evolution of the bed profile and the water height with $\mathrm{A}=0.001$ at time $\mathrm{t}=230000 \mathrm{~s}$ is depicted in Figure 4. The results with $\mathrm{A}=1$ at time $\mathrm{t}=624 \mathrm{~s}$ are included in Figure 3 . The proposed discontinuous Galerkin method performs very satisfactorily for this nonlinear coupled problem since it does not diffuse the moving bed fronts and no spurious oscillations have been detected near steep gradients of the flow field in the computational domain. In this test example, the degree of the polynomial approximation is fixed to $N=6$. 

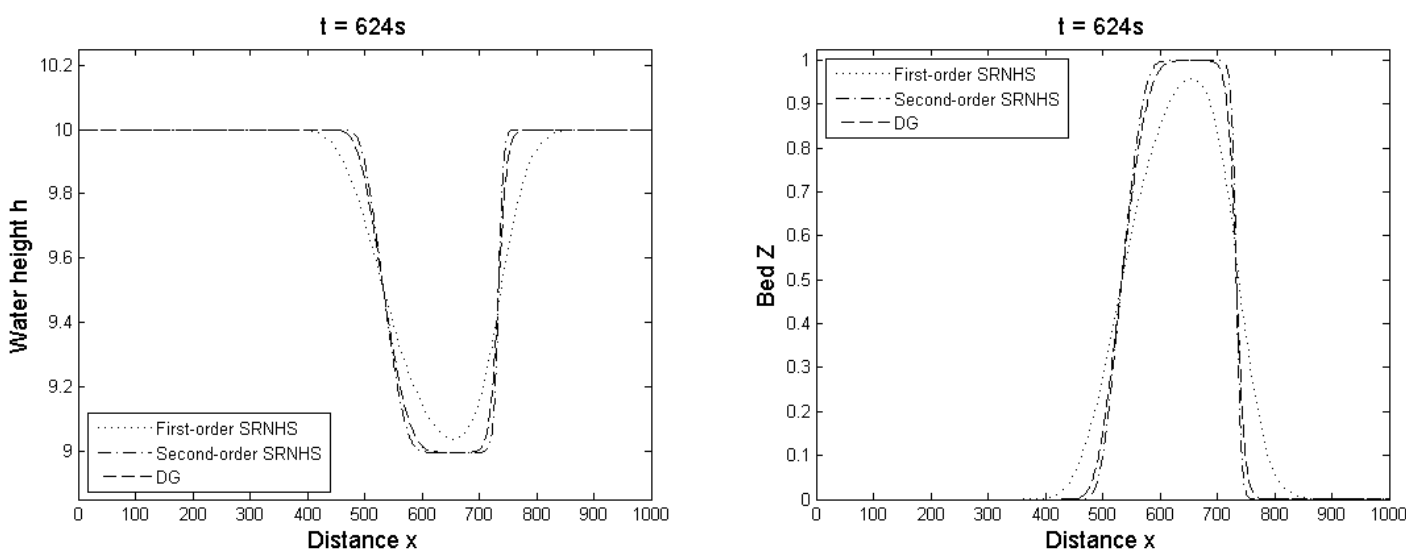

Fig 3: Water height (left) and bed-load (right) with $A=1$ using coupled approach.
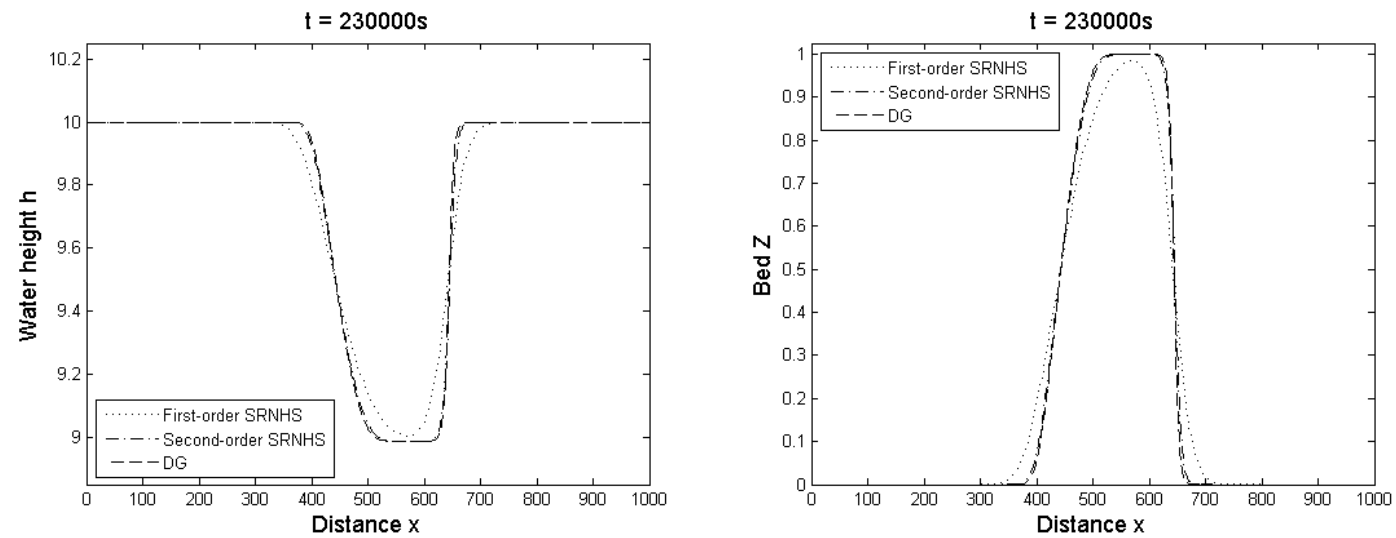

Fig 4: Water height (left) and bed-load (right) with $A=0.001$ using decoupled approach.

\subsection{Test 3}

This test was presented in [19] as to present the case of a sediment bore that interacts quickly with the water flow over a step. To obtain this situation $A=1$ and $\varepsilon=0.2$ The initial riverbed is given by

$$
\mathrm{Z}(0, x)=\frac{1}{1+\exp ((x-400) / 5 \pi)} .
$$

In Figure 5, we present the numerical results obtained using different orders of polynomial approximation. It is clear that for $N=2$ and $N=4$, the numerical diffusion is very

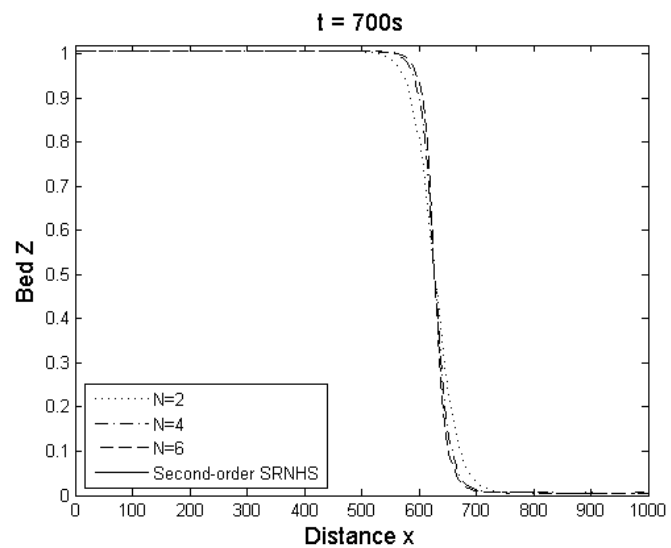

pronounced in the numerical solutions. This excessive numerical dissipation has been successfully removed in the bed-load and water height by increasing the values of $N$. The bores propagate downstream, increasing their steepness during their evolution in space and time. For this problem again the DG scheme produced similar smooth results behaving very well near the bore and compare well with the results presented in [19]. Needless to mention that increasing the degree $N$ results in an increase of the computational cost in the DG method. For then considered degree of the polynomial approximation $N=2, N=4$ and $N=6$ the required CPU time in the DG method is $8.844,32.372$ and 80.833 minutes, respectively.

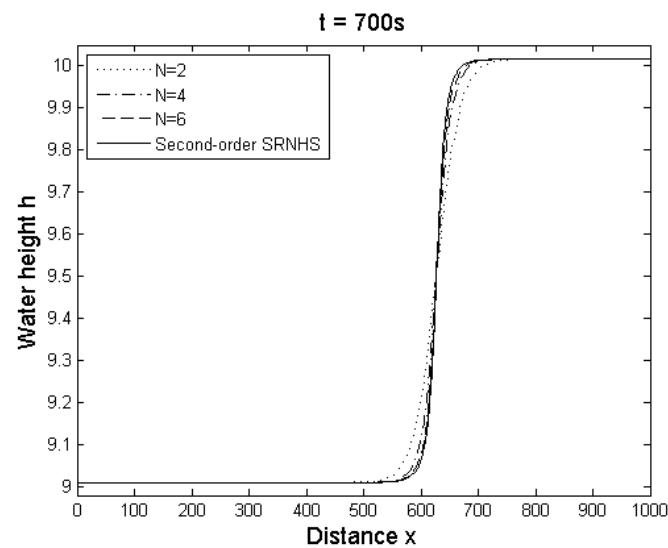

Fig 5: Water height (left) and bed-load (right) with $A=1$ using coupled approach. 


\subsection{Test 4}

Our final test example [8] is the problem of a sediment transport with backward/forward step. Here the initial bed is defined as

$$
\mathrm{Z}(0, x)= \begin{cases}1, & \text { if } \quad 0 \leq x \leq 96, \\ \frac{-x+181}{85} & \text { if } \quad 96 \leq x \leq 181 \\ 0, & \text { if } \quad 181 \leq x \leq 638 \\ \frac{x-638}{85} & \text { if } \quad 638 \leq x \leq 723 \\ 1, & \text { if } 723 \leq x \leq 1000,\end{cases}
$$

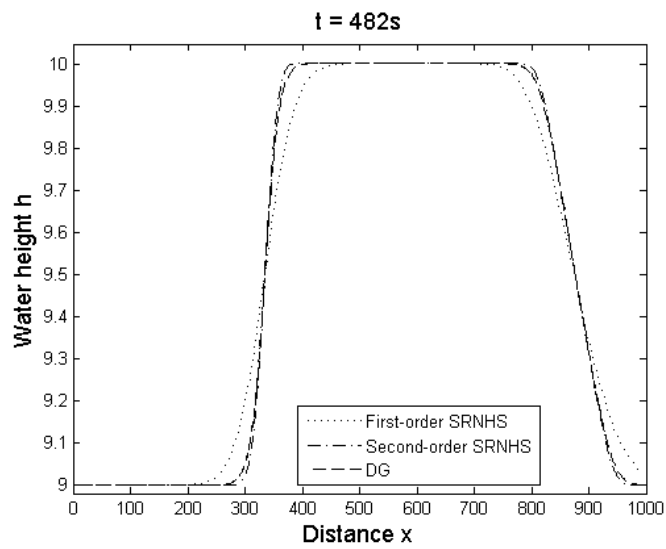

A similar test example was studied in [5] for sedimentary flows. We display in Figure 6 the bed-load and the water height with $A=1$ at $t=482 \mathrm{~s}$. Figure 7 reports the obtained results with $A=0.001$ at time $t=250000 \mathrm{~s}$. In both case, we run the code with $N=4$. As in the previous test examples, the DG formulation has calculated correctly the bed-load and the water height.

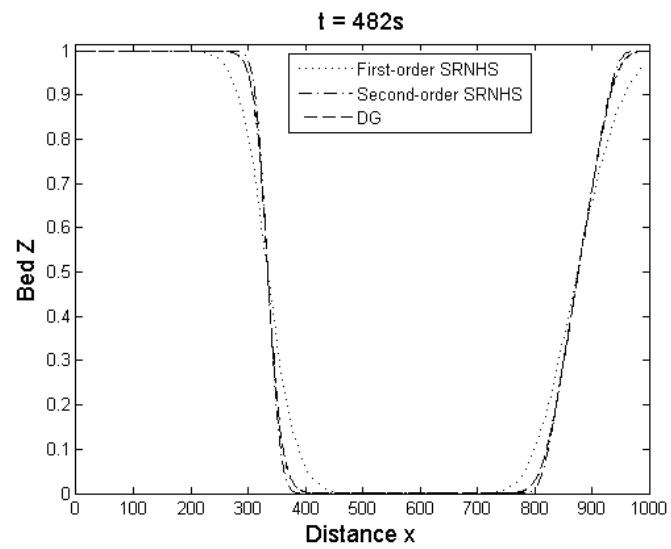

Fig 6: Water height (left) and bed-load (right) with $A=1$ using coupled approach.
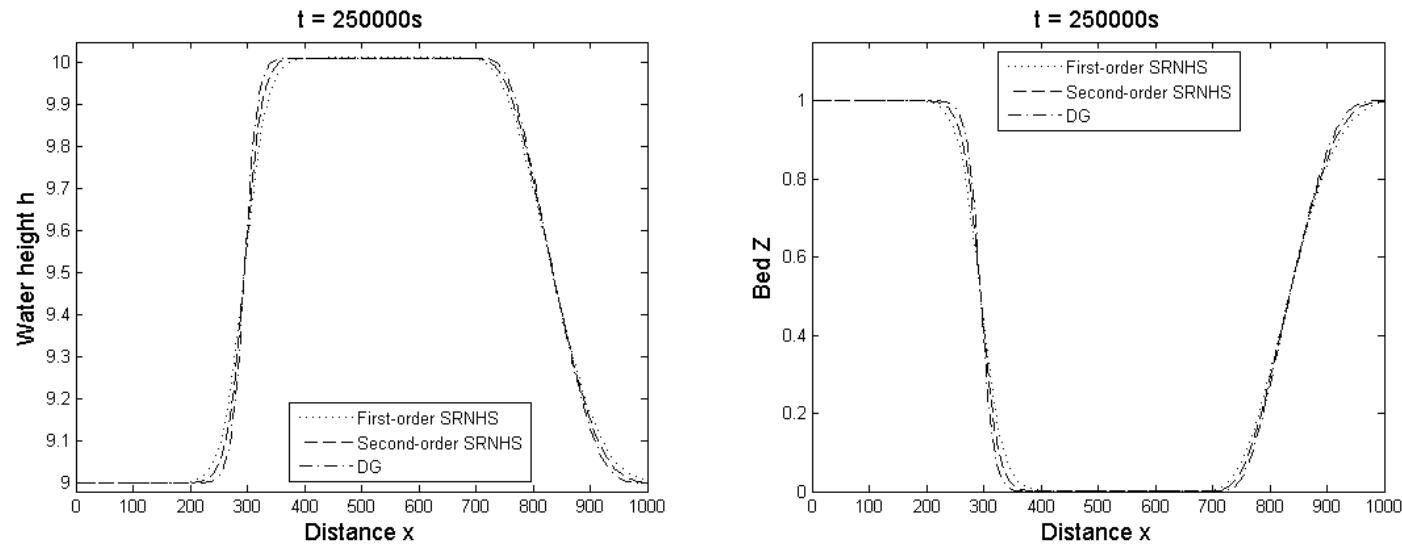

Fig 7: Water height (left) and bed-load (right) with $A=0.001$ using decoupled approach.

\section{CONCLUSION}

In this work we have presented a discontinuous Galerkin finite-element method to the bed-level sediment transport, in one space dimension. The mathematical models presented are relatively simple but not trivial to solve and present a relatively ideal but conceptually important situation in morphodynamic modeling. The DG method has been tested for a class of sediment transport problems with different bed interaction and flow regimes. The performance of DG scheme is very attractive since the computed solution remains, stable, monotone and reasonably accurate even on coarse grids. The obtained results indicate good shock resolution with high accuracy in smooth regions and without any nonphysical oscillations near the shock areas. The numerical comparison reported in $[6,8,7]$ for the same sediment transport problems support this statement. An extension of the scheme for twodimensional morphodynamic problems is under consideration.

\section{ACKNOWLEDGMENTS}

Financial support provided by MULIT and MHYCOF projects is gratefully acknowledged.

\section{REFERENCES}

[1] Grass AJ. Sediment transport by waves and currents. SERC London Cent. Mar. Technol. Report No: FL29, 1981.

[2] Y. Bazilevs and TJR. Hughes. Weak imposition of dirichlet boundary conditions in fluid mechanics. Computers \& Fluids, in press, 36:12-26, 2007. 
[3] Bernini A. Caleffi V, Valiani A. High-order balanced cweno scheme for movable bed shallow water equations. Advances in Water Resources, 30:730$741,2007$.

[4] B. Cockburn, G. E. Karniadakis, and C.-W. Shu (eds.). Discontinuous Galerkin methods. Theory, computation and applications. Lecture Notes in Computational Science and Engineering, 11. SpringerVerlag, Berlin, 2000.

[5] Holz K.P. Crotogino A. Numerical movable-bed models for practical engineering. Applied Mathematical Modelling, 8:45-49, 1984.

[6] Papoglou I. DelisA.I. Relaxation approximation to bedload sediment transport. J. Comput. Appl. Math., 213:521-546, 2008

[7] M. Seaid, F. Benkhaldoun, S. Sahmim. "Solution of the sediment transport equations using a finite volume method based on sign matrix". SIAM J. Sci. Comp, Vol. 31, No. 4:2866-2889, 2009.

[8] M. Seaid, F. Benkhaldoun, S. Sahmim. Mathematical development and verification of a finite volume model for morphodynamic flow applications. accepted in Advances in Applied Mathematics and Mechanics, 2011.

[9] S. Gottlieb, C.W. Shu, and E. Tadmor. Strong stability preserving high order time integration methods. SIAM Rev., 43:89-112, 2001.

[10] J.S. Hesthaven and T. Warburton. High-order nodal methods on unstructured grids. I. time-domain solution of maxwells equations. J Comp Phys, 181(1):186-221, 2002.

[11] J. Hudson. Numerical techniques for morphodynamic modelling. Ph.D. Thesis, University of Reading, 2001.

[12] Sweby P.K. Hudson, J. Formulations for numerically approximating hyperbolic systems governing sediment transport. J. Sci. Comput., 19:225-252, 2003.
[13] TJR. Hughes, G. Scovazzi, PB. Bochev, and A. Buffa. A multiscale discontin- uous Galerkin method with the computational structure of a continuous Galerkin method. Computer Methods in Applied Mechanics and Engineering, 195:2761-2787, 2006.

[14] N. Dodd T. Chesher A. Cooper J. Hudson, J. Damgaard. Numerical approaches for $1 \mathrm{~d}$ morphodynamic modelling. Coastal Eng., 52:691-707, 2005.

[15] C. W. Shu J. Qiu, B. C. Khoo. A numerical study for the performance of the rungekutta discontinuous galerkin method based on different numerical fluxes. J. Comput. Phys., 212:540-565, 2006.

[16] A. Verway J.A. Cunge, F.M. Holly. Practical Aspects of Computational River Hy- draulics. Pitman, London, 1980.

[17] R.J. Leveque. Finite Volume Methods for Hyperbolic Problems. Cambridge Univer- sity Press, Cambridge, 2002.

[18] Shao Z. Long W, Kirby J.T. A numerical scheme for morphological bed level calculations. Coastal Engineering, 55:167-180, 2008.

[19] L. Sopta N. Crnjaric-Zic, S. Vukovic. Extension of eno and weno schemes to one-dimensional sediment transport equations. Comput. Fluids, 33:31-56, 2004

[20] Roe P.L. Approximate riemann solvers, parameter vectors and difference schemes. J. Comp. Physics. 43:357-372, 1981 .

[21] Hogg A.J. Pritchard D. On sediment transport under dam-break flow. J. FluidMech., 473:265-274, 2002.

[22] Fraccarollo L. Rosatti G. A well-balanced approach for flows over mobile-bed with high sediment-transport. J. Comput. Physics, 220:312-338, 2006.

[23] C.W. Shu. Total variation diminishing time discretizations. SIAM J. Sci. Stat. Comput., 9:10731084, 1988 